\title{
Integration of LCoS-SLM and LabVIEW based software to simulate fundamental optics, wave optics, and Fourier optics
}

Bo-Han Lyu, Chen Wang, Chun-Wei Tsai

Bo-Han Lyu, Chen Wang, Chun-Wei Tsai, "Integration of LCoS-SLM and LabVIEW based software to simulate fundamental optics, wave optics, and Fourier optics," Proc. SPIE 10452, 14th Conference on Education and Training in Optics and Photonics: ETOP 2017, 104521P (16 August 2017); doi: $10.1117 / 12.2257067$

Event: 14th Conference on Education and Training in Optics and Photonics, ETOP 2017, 2017, Hangzhou, China 


\title{
Integration of LCoS-SLM and LabVIEW Based Software to Simulate Fundamental Optics, Wave Optics, and Fourier Optics
}

\author{
Bo-Han Lyu, Chen Wang, and Chun-Wei Tsai* \\ Jasper Display Corp. (JDC), 7F-16, No. 81, Shui-Li Rd., Hsinchu 30059, Taiwan
}

\begin{abstract}
Jasper Display Corp. (JDC) offer high reflectivity, high resolution Liquid Crystal on Silicon - Spatial Light Modulator (LCoS-SLM) which include an associated controller ASIC and LabVIEW based modulation software. Based on this LCoS-SLM, also called Education Kit (EDK), we provide a training platform which includes a series of optical theory and experiments to university students. This EDK not only provides a LabVIEW based operation software to produce Computer Generated Holograms (CGH) to generate some basic diffraction image or holographic image, but also provides simulation software to verity the experiment results simultaneously. However, we believe that a robust LCoSSLM, operation software, simulation software, training system, and training course can help students to study the fundamental optics, wave optics, and Fourier optics more easily. Based on these fundamental knowledges, they could develop their unique skills and create their new innovations on the optoelectronic application in the future.
\end{abstract}

Keywords: Liquid Crystal on Silicon (LCoS), Spatial Light Modulator (SLM), Education Kit (EDK), LabVIEW, Optical Simulation, Optics Education, Fourier Optics, Computer Generated Holograms (CGH)

\section{INTRODUCTION}

Liquid Crystal on Silicon-Spatial Light Modulator (LCoS-SLM) has been widely used on various applications, such as adaptive optics [1-3], holographic display [4-5], microscopy [6-7], beam shaping [8], beam steering [9], optical communication [10], diffractive optical element (DOE) [11], semiconductor equipment [12], and 3D metrology [13]. The major reason is that LCoS-SLM is a useful and powerful optical instrument to modulate the optical wavefront dynamically. By adjusting the driving voltage, the degree of twist of liquid crystal molecules could be changed and tilted. The light will have phase retardation when it passes through liquid crystal layer. Generally, LCoS-SLM is capable of accepting a video signal and converting it into a holographic image. The video signal, also called computer generated holograms $(\mathrm{CGH})$, is one method to generate holographic interference patterns digitally. There are three steps to generate CGH as below, (1) computing the virtual scattered wavefront, (2) encoding the interference pattern, and (3) reconstructing the holographic image. There are two type of LCoS-SLM, such as transmissive type and reflective type, respectively. The LCoS-SLM JDC offer is an electrically addressed reflective type. The reflective type LCoS-SLM typically has more efficient light usage and less annoying diffraction by high aperture ratio. In addition, we also offer high resolution and small pixel pitch LCoS-SLM which includes an associated controller ASIC and LabVIEW based modulation software. We also offer a training platform which includes a series of optical theory and experimental course. Through the promotion of LCoS-SLM in university, we expect that these university students could develop their unique skills and spur innovations for future optoelectronic applications through our training program. There are mainly three topics in our training program or experimental course. The first topic is "Fundamentals of LCoS-SLM" which includes polarization, jones matrix, and uniaxial crystal theory. The second topic is "Wave Optics" which includes interference, diffraction, and dispersion phenomenon. The third topic is "Fourier Optics" which includes spatial frequency, optical spectrum, Fresnel diffraction, and Fraunhofer diffraction. But it is not limited. Teachers could design their own experiments and then implement them on EDK.

*chunwei.tsai@jasperdisplay.com; phone 886-3-575-2768; fax 886-3-575-2628; www.jasperdisplay.com

14th Conference on Education and Training in Optics and Photonics: ETOP 2017, edited by Xu Liu,

Xi-Cheng Zhang, Proc. of SPIE Vol. 10452, 104521P · C 2017 ICO, IEEE, OSA, SPIE

CCC code: $0277-786 X / 17 / \$ 18 \cdot$ doi: $10.1117 / 12.2257067$

Proc. of SPIE Vol. 10452 104521P-1 
Figure 1 (a) shows the basic experiment setup diagram based on LCoS-SLM (EDK), and Figure 1 (b) shows the experiment setup photo of EDK. The first part of our optical system (gray) from the coherent light source to collimating lens is collimating system which produces a plane wave to illuminate the LCoS-SLM. The second part is polarization system (red), two polarizers constrain the input and output polarization state to switch SLM between amplitude modulator and phase modulator. The last part is an imaging system (blue), the imaging lens will form LCoS-SLM image onto screen or CCD. Our Educational Kit (EDK) for LCoS-SLM mainly uses these three parts to compose ten experiments.

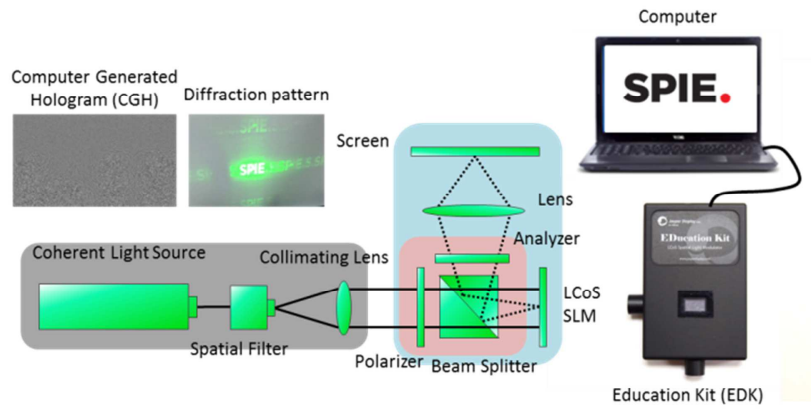

(a)

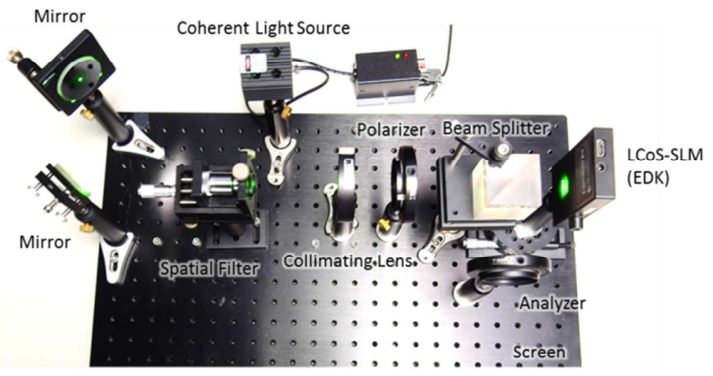

(b)

Figure 1. (a) Basic experiment setup diagram based on LCoS-SLM (EDK), and (b) Experiment setup photo based on EDK.

\section{PREVIOUS WORK - SLM BASED EDUCATION KIT}

Spatial Light Modulator (SLM) is a powerful active optical component to change the optical wavefront by electrical signal and had been widely investigated for many applications. We use LCoS (Liquid Crystal on Silicon) which has high reflectivity and spatial resolution as main component of SLM educational kit [14], and present a series of experiment to let students know the background theory of SLM. There are mainly three topics in our training program or experimental course. The first topic is "Fundamentals of LCoS-SLM," the second topic is "Wave Optics," and the third topic is "Fourier Optics."

\subsection{Fundamentals of LCoS-SLM}

LCoS-SLM has pixelated structure as micro display. The coherent light source would be diffracted to vertical and horizontal direction when the coherence light source illuminates the pixel structure of LCoS-SLM. The polarization state is changed by the anisotropic characteristic of liquid crystal. Regarding this characteristic of liquid crystal, LCoS-SLM can modulate the amplitude or phase of the light. From the first topic "Fundamentals of LCoS-SLM," we would like to let the university students understand these characteristics of LCOS-SLM and realize how to modulate the basic wavefront by using LCoS-SLM. However, the first topic includes the polarization, jones matrix, and uniaxial crystal theory. Figure 2 shows the fundamental optics based on LCoS-SLM (EDK). The pixelated structure, amplitude modulation, phase modulation, and wavefront modulation experiments are shown as Figure 2 (a)-(d), respectively. 

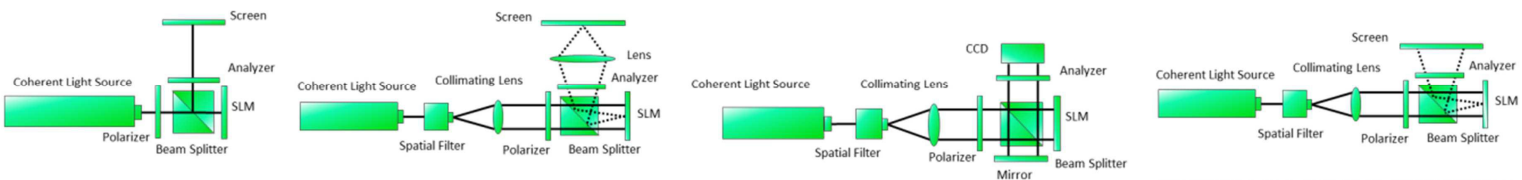

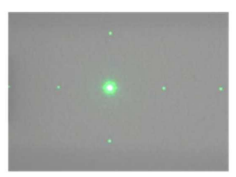

Diffracted light spot

(a)

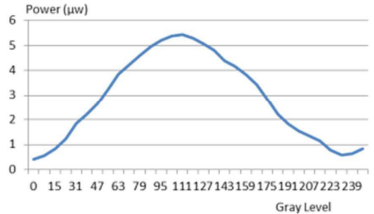

(b)

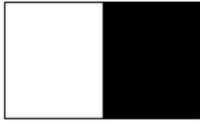

Input pattern

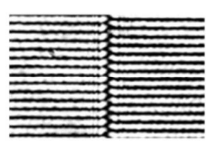

(c)

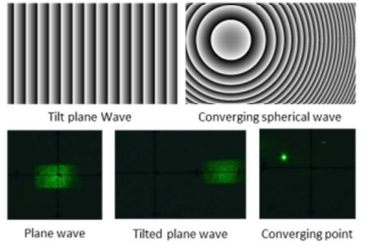

(d)

Figure 2. Fundamental optics based on LCoS-SLM (EDK), (a) pixelated structure, (b) amplitude modulation, (c) phase modulation; and (d) wavefront modulation.

\subsection{Wave Optics}

In this second topic "Wave Optics," LCoS-SLM will be applied to traditional optical experiments by providing the phase grating and amplitude mask. We expect to let the university students know how to apply the LCoS-SLM into the traditional experiments. However, the second topic includes interference, diffraction, and dispersion phenomenon. Figure 3 shows the wave optics based on LCoS-SLM (EDK). The diffraction and interference, the Michelson interferometer, and the dispersion experiments are shown as Figure 3 (a)-(c), respectively.

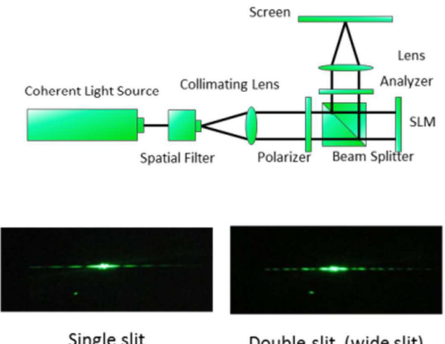

(a)
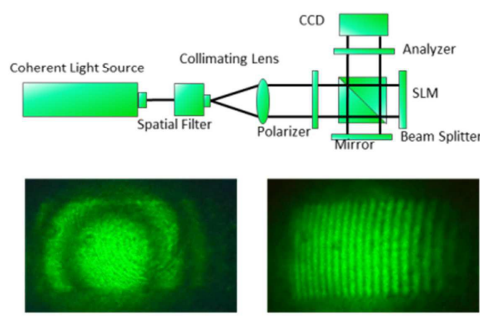

Spherical and plane wave

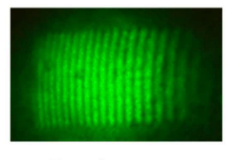

Two plane wave

(b)

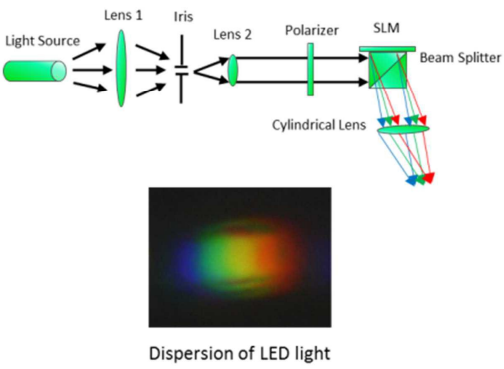

(c)

Figure 3. Wave optics based on LCoS-SLM (EDK), (a) diffraction and interference, (b) Michelson interferometer, and (c) dispersion.

\subsection{Fourier Optics}

The third topic is "Fourier Optics" which includes spatial frequency, optical spectrum, Fresnel diffraction, and Fraunhofer diffraction. Figure 4 Shows the Fourier optics based on LCoS-SLM (EDK). The signal processing, Talbot mage, and phase shift digital holography (PSDH) are shown as Figure 4 (a)-(c), respectively. LCoS-SLM has these properties of pixelated structure, high aperture ratio, and smaller pixel size. Hence, the LCoS-SLM could be a good solution to perform the Fourier experiments such as Fresnel and Fraunhofer diffraction. Most important of all, if we want to get a high reflective and clear diffraction results, we need to use a digital pattern and to make these pixel size as small as the wavelength range. 

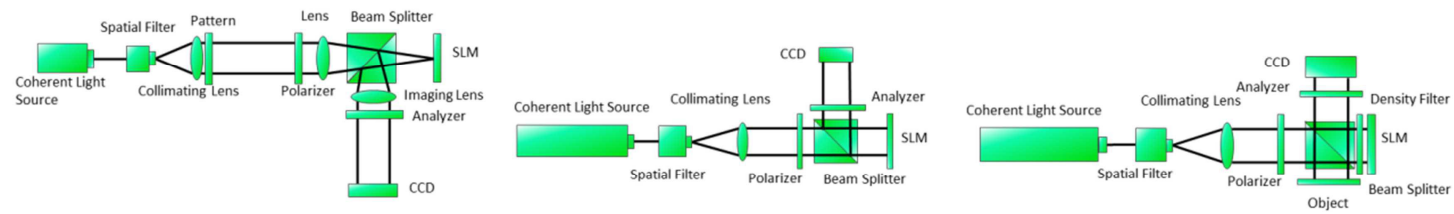

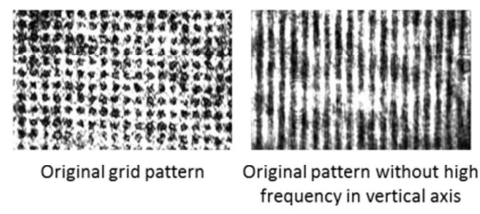

(a)

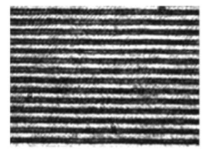

Talbot image

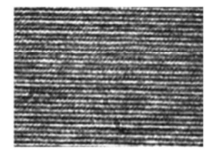

Talbot subimage

(b)

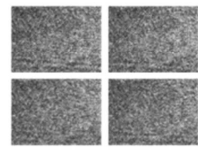

One set of interference

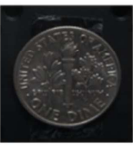

Original image

(c)

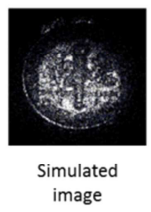

Figure 4. Fourier optics based on LCoS-SLM, (a) signal processing, (b) Talbot mage, and (c) phase shift digital holography..

\section{NEW FUNCTION AND NEW SIMULATION SOFTWARE ON LCOS-SLM (EDK)}

This EDK not only provides a LabVIEW based operation software to produce CGH to generate some basic diffraction image or holographic image, but also provides simulation software to verity the experiment results simultaneously. Our LabVIEW based software also can simulate fundamental optics, wave optics, and Fourier optics. This simulation software not just helps students to realize the theory of Jones matrix and the relationship among of polarizer, retarder, and analyzer, but can simulate the interference, diffraction, and dispersion phenomenon. We also can use this simulation software to simulate the optical pattern and optical wavefront dynamically. However, we believe that a robust LCoSSLM, operation software, simulation software, training system, and training course can help students to study the fundamental optics, wave optics, and Fourier optics more easily. Based on these fundamental knowledges, they could develop their unique skills and create their new innovations on the optoelectronic application in the future.

\subsection{Pixelated Structure of LCoS-SLM}

The objective of this experiment is to investigate how the basic structure of the SLM affects the behaviors of the light, by projecting a light wave onto an LCoS-SLM that is not receiving electrical signals. We will learn how to observe the maximum diffraction angle, diffraction efficiency, and the changes in the polarization state; then, use these observations to calculate the basic parameters of the LCoS-SLM, such as its pixel size, aperture ratio, and alignment angle. Figure 5 (a) shows the Graphical User Interface (GUI) of pixelated structure. This EDK software is LabVIEW based and provide multi-function and easy-to-use modulation. Figure 5 (b) shows the GUI of calculating pixel size. By Fourier series analysis, a periodical structure could be expressed by the following equation

$$
f(x)=\sum_{-\infty}^{\infty} A_{n} e^{j 2 \pi \frac{n}{D} x}
$$

where

$$
A_{n}=\frac{1}{D} \int_{-D / 2}^{D / 2} f(x) e^{-j 2 \pi \frac{n}{D} x} d x
$$

$D$ is the period of the structure, and $n$ is the $n_{t h}$ order of diffraction. From the equation above, the original equation can be broken down into several exponential functions with different weights, which represent several diffracted light beams of different orders, with the diffraction angle $\theta$ as,

$$
\theta=\sin ^{-1}\left(\frac{n \lambda}{D}\right)
$$

where $\lambda$ is the wavelength of the coherent light source. Under the condition of paraxial approximation, the diffraction angle will increase proportionally. Thus, using the above equation, we can calculate the pixel period $D$ by, first, measuring the diffraction angle, then, substituting it with the wavelength of the light. Under the condition of a fixed wavelength, a larger diffraction angle requires a smaller pixel period. This is why SLMs need to be made smaller with their dimensions made closer to the wavelength of the light. 
From the above conclusion, we know that the distribution curve of the intensity of diffracted light is a sinc function as below,

where

$$
\frac{I_{1}}{I_{0}}=\left[\operatorname{sinc}\left(\frac{d}{D}\right)\right]^{2}
$$

$$
\operatorname{sinc}(x)=\frac{\sin (\pi x)}{\pi x}
$$

$I_{0}$ is the intensity of zero order and $I_{l}$ is the intensity of first order. $d$ is the aperture size and also be the pixel size. $D$ is the period of the structure and also be the pixel pitch. $x$ is the coefficient $\frac{d}{D}$ of the sinc function.

By calculating the diffraction angle, we can also use the following equation based on our experiment setup,

$$
\theta=\tan ^{-1}\left(\frac{x_{1}}{d_{1}+d_{2}}\right)
$$

where $x_{l}$ is the distance between zero order to first order, $d_{l}$ is the distance between LCoS-SLM to the beam splitter, and $d_{2}$ is the distance between the beam splitter to the screen.

Figure 6 (a) shows the experiment setup photo of pixelated structure. Due to the pixelated structure of LCoS-SLM, a coherent light source $(532 \mathrm{~nm})$ normally incident on LCoS-SLM and then the light will be reflected back with many diffraction orders as shown in Figure 6 (b). Based on this software of calculating pixel size, the students could fill the blanks of parameters such as SLM to Beam Splitter $\left(d_{1}\right)$, Beam Splitter to Screen $\left(d_{2}\right)$, Diffraction angle $(\theta)$, Order of Diffraction $(n)$, Wavelength of the coherent light source $(\lambda)$, zero order Power $\left(I_{0}\right)$, and first order Power $\left(I_{l}\right)$. After that, the program can help the students to calculate the Pitch Size $(D)$ and Pixel Size $(d)$. For example, the distance of diffracted light spots between zero order to first order $\left(x_{I}\right)$ we measured is $2.9 \mathrm{~cm}$. The distance between LCoS-SLM to the beam splitter $\left(d_{1}\right)$ and the distance between the beam splitter to the screen $\left(d_{2}\right)$ are $5.4 \mathrm{~cm}$ and $30 \mathrm{~cm}$, respectively. The intensity of zero order and first order is $544.2 \mu \mathrm{W}$ and $1.7 \mu \mathrm{W}$, respectively. From the above parameters of our LCoS-SLM, the diffraction angle is 0.08174 rad and intensity ratio of first order to zero order is 0.003124 , and we can obtain that the period $(D)$ is $6.516 \mu \mathrm{m}$, the aperture size $(d)$ is $6.169 \mu \mathrm{m}$, and the aperture ratio is $90.2 \%$ from this experiment.

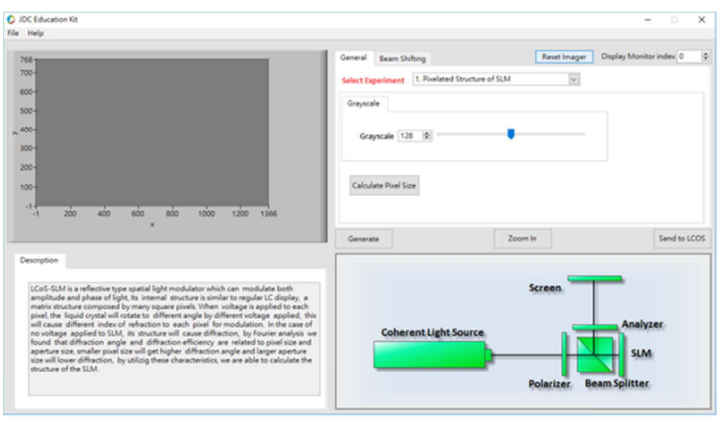

(a)

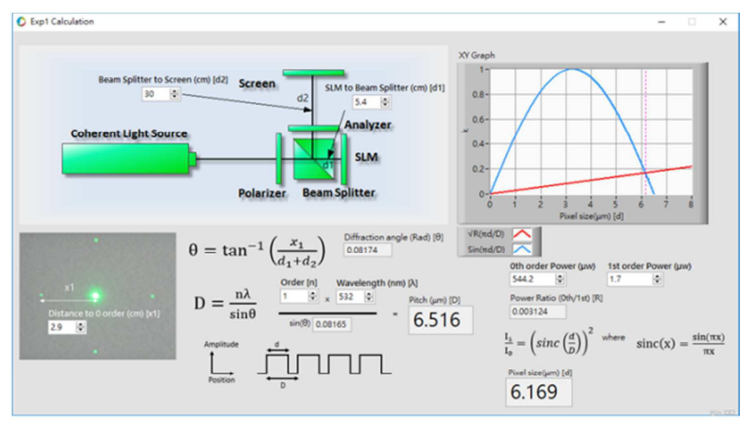

(b)

Figure 5. (a) LabVIEW based software GUI of pixelated structure, and (b) GUI of calculating pixel size. 


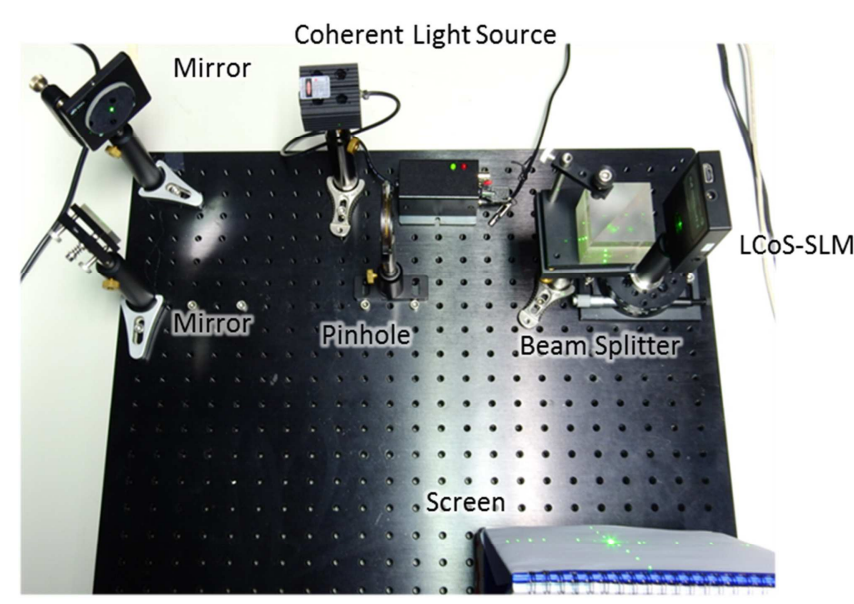

(a)

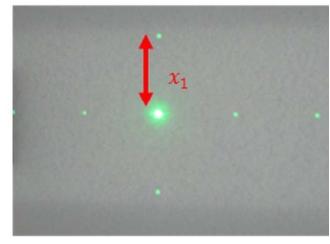

(b)

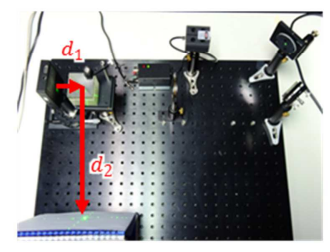

(c)

Figure 6. (a) Experiment setup photo of pixelated structure, (b) The distance of diffracted light spots $\left(x_{1}\right)$ between zero order to first order, and (c) The measurement distance $\left(d_{1}+d_{2}\right)$ from LCoS-SLM to the screen.

\subsection{Amplitude Modulation}

Regarding amplitude modulation experiment, we need to look for the alignment angle of LCoS-SLM. We also need to adjust the angles of the polarizer and the analyzer to convert LCoS-SLM into an amplitude modulator; then we can create a simple projection system. In this experiment, students will learn how to use LCoS-SLM as an amplitude modulator. The LabVIEW based software GUI of amplitude modulation is shown as Figure 7 (a). In addition, the students can use our Amplitude Modulation Simulation software to simulate the phenomenon of amplitude modulation. The GUI of amplitude modulation simulation is shown as Figure 7 (b). About this software of amplitude modulation simulation, the students could fill the blank parameters of Polarizer Transmission Axis, Liquid Crystal Optical Axis, Retarder Retardation, and Analyzer Transmission Axis. The Polarizer Transmission Axis is the transmittance direction of the input polarization light. Typically, it is called Polarizer direction. Liquid Crystal Optical Axis is the direction of uniaxial retarder slow axis. Retarder Retardation is the retardation of the retarder. Analyzer Transmission is the transmittance direction of the output polarization light. Typically, it is called Analyzer direction. The experiment setup photo is shown in Figure 8 (a), a plane wave passes through LCoS-SLM with two polarizer controlling input and output polarization state, an imaging lens behind the LCoS-SLM forms the SLM image onto screen. The transmittance of this system [15-17] is

$$
\mathrm{T}=\cos ^{2} \chi-\sin 2(\beta-\chi) \sin (2 \beta) \sin ^{2}\left[\frac{\delta}{2}\right]
$$

where $\chi$ is the angle between polarizer and analyzer, $\beta$ is the angle between the polarizer and LC director, and $\delta$ is the phase retardation related to voltage applied. With a cross polarized configuration which $\chi$ equal to 90 degree and $\beta$ equals to 45 degree. The transmittance becomes

$$
\mathrm{T}_{\mathrm{A}}=\sin ^{2}\left[\frac{\delta}{2}\right] \text {. }
$$

We can get an amplitude modulator with sinusoidal response by this configuration. Figure 8 (b) shows the sinusoidal intensity variation by different grayscale input from computer. 


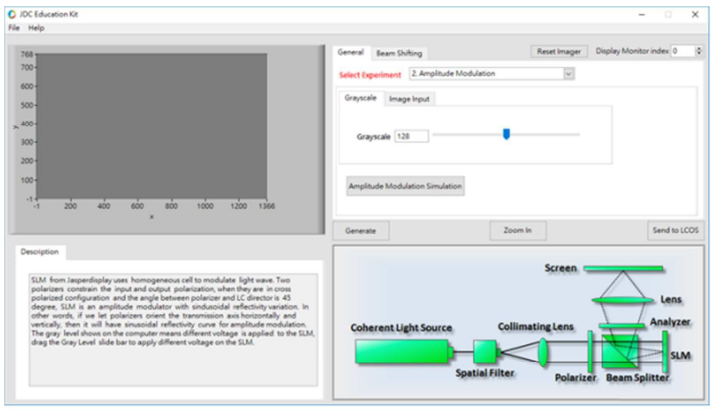

(a)

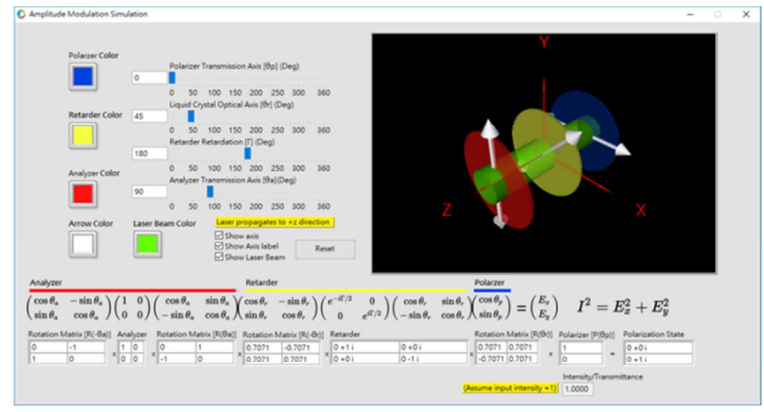

(b)

Figure 7. (a) LabVIEW based software GUI of amplitude modulation, and (b) GUI of amplitude modulation simulation.

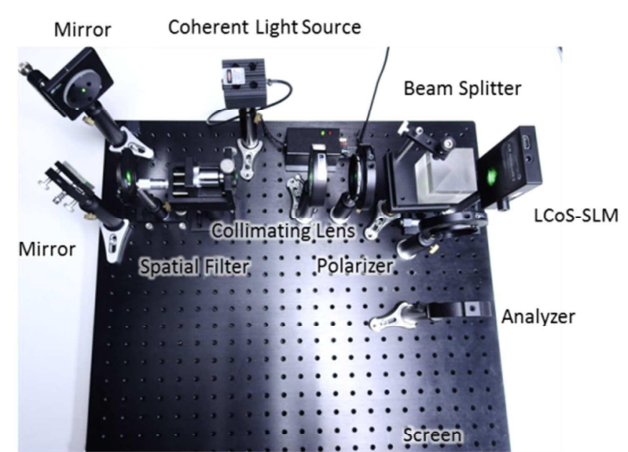

(a)

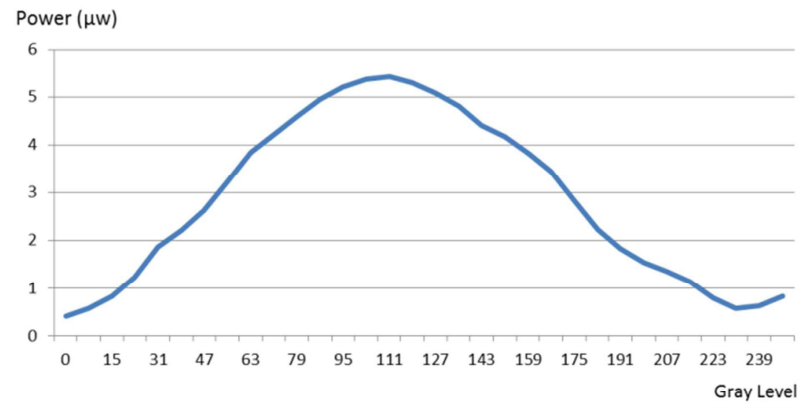

(b)

Figure 8. (a) Experiment setup photo of amplitude modulation, and (b) Intensity variation at different gray level input.

\subsection{Phase Modulation}

Regarding phase modulation experiment, we adjust the angles of the polarizer and the analyzer to convert the spatial light modulator into a phase modulator. In addition, we use an interferometer to measure its phase changes. The LabVIEW based software GUI of phase modulation is shown as Figure 9 (a). In addition, the students can use our Phase Modulation Simulation software to simulate the phenomenon of phase modulation. The GUI of phase modulation simulation is shown as Figure 9 (b). About this software of phase modulation simulation, the students could fill the wavelength of the coherent light source, pixel size, and homogeneous patterns with different gray level. Then, the students can simulate the phenomenon at different diffraction distance, different focal length, and different $x$ or $y$ direction offset. In phase modulation experiment, we use the same optical experiment setup as amplitude modulation experiment but change the polarizer and analyzer direction to align with LC director. It means $\chi=\beta=0$. As a result, the transmittance is always 1, there is no amplitude variation at this configuration. Only phase varies with input signal. Moreover, we would like to examine the phase modulation capability by the interference. Therefore, we add one more mirror for offering the reference light in this experiment. The experiment setup photo of phase modulation experiment is shown in Figure 10 (a). We adjust the tilt angle of mirror to get a horizontal interference and then put a black/white pattern to the LCoS-SLM. Each gray level represents different phase difference. The white parts will gradually changes its gray level from 0 to 255 , and the interference pattern will be shifted as equation below

$$
I=2 I_{0}[1+\cos (k \sin \theta y-\phi)]
$$

where $k$ is the wavenumber, $\theta$ is the angle between two plane wave reflected from LCoS-SLM and mirror, $\phi$ is the phase difference between these two waves. The phase shift is in proportion to interference shift, thus we can measure the phase difference by the shifted interference. The phase difference results at different gray level input are shown as Figure 10 (b). 


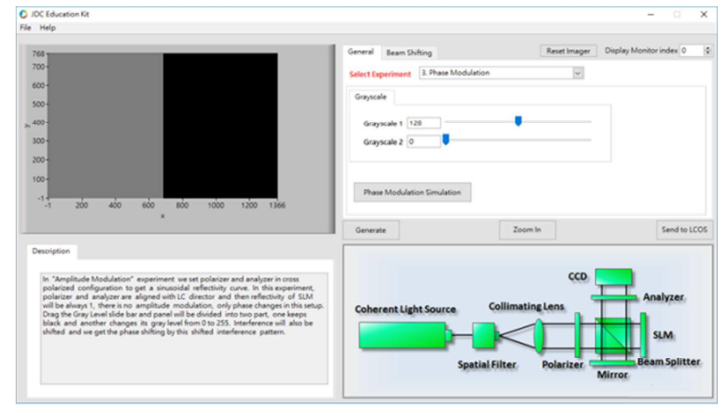

(a)

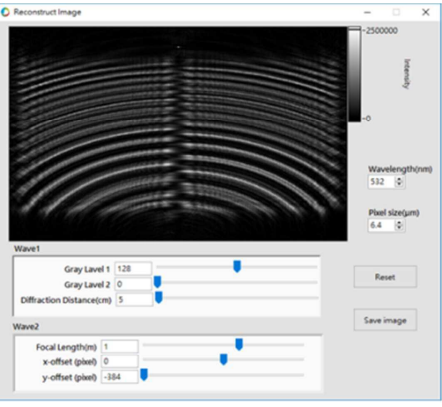

(b)

Figure 9. (a) LabVIEW based software GUI of phase modulation, and (b) GUI of phase modulation simulation.

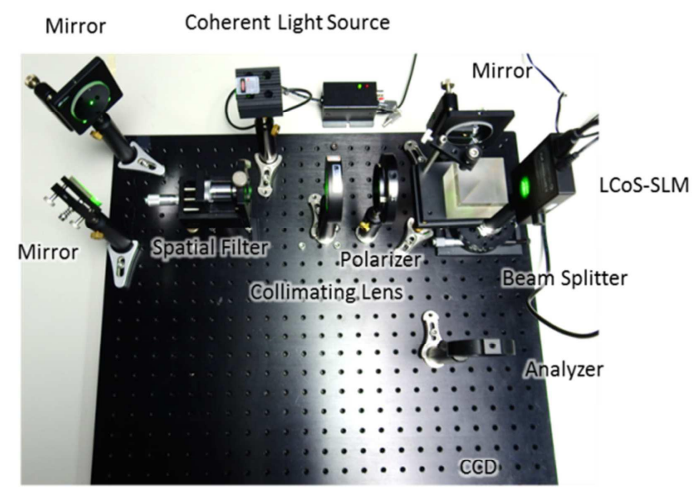

(a)

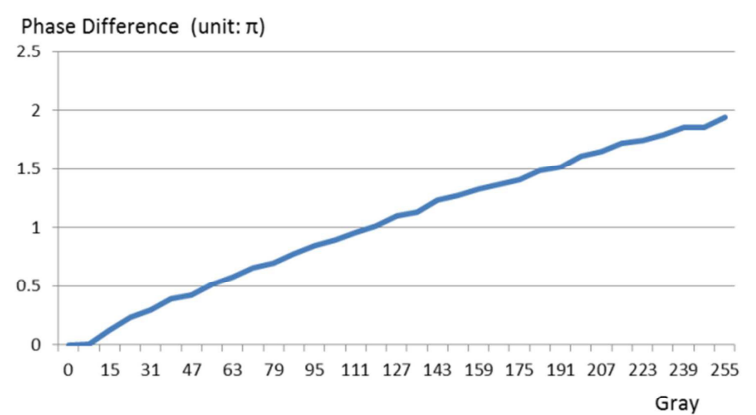

(b)

Figure 10. (a) Experiment setup photo of phase modulation, and (b) Phase difference at different gray level input.

\subsection{Wavefront Modulation}

The objective of this experiment is to create plane waves, spherical waves, and cylindrical waves that travel along different directions by adjusting the wavefronts of phase modulator. In addition, we would like to learn about the limits of the diffraction angle of a phase modulator. In this experiment, the students have known how to use SLM as a phase modulator in previous experiment, and they will learn how to modulate the incident plane wave to converge, diverge, and orientates to other directions. The plane wavefront, spherical wavefront, and cylindrical wavefront in the LCoS-SLM plane ( $x-y$ plane) can be expressed as following equations

$$
\begin{gathered}
\text { Plane wave: } e^{i \frac{2 \pi}{\lambda} x \sin \theta} \\
\text { Spherical wave: } e^{i \frac{\pi}{\lambda z_{0}}\left(x^{2}+y^{2}\right)} \\
\text { Cylindrical wave (phase only changes along the } y \text {-axis): } e^{i \frac{\pi}{\lambda z_{0}} y^{2}}
\end{gathered}
$$

Where $\theta$ is the angle between $z$ axis and propagation direction, $z_{0}$ is the distance from diverging or converging point to the LCoS-SLM plane. The LabVIEW based software GUI of wavefront modulation is shown as Figure 11 (a). In addition, the students can use our Wavefront Modulation Simulation software to simulate the phenomenon of wavefront modulation. The GUI of wavefront modulation simulation is shown as Figure 11 (b). Regarding this software of wavefront modulation simulation, the students could fill the wavelength of the coherent light source, and pixel size. Then, the students can simulate the phenomenon to observe the result of different parameters by changing the observe distance, and the wavefront type. The wavefront type includes plane wave, spherical wave, and cylindrical wave. The experiment setup photo of wavefront modulation experiment is shown in Figure 12 (a). We can directly get the phase information by 
substituting pixels position $(x, y)$ data of SLM, each pixel will have its phase information and each phase data corresponds to gray level input from computer, the resulting plot are shown in Figure 12 (b).

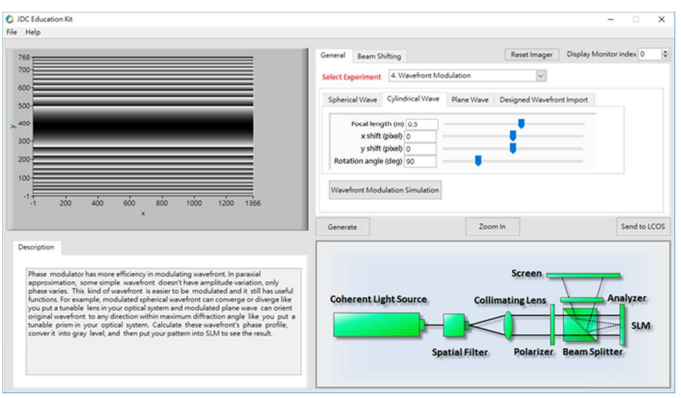

(a)

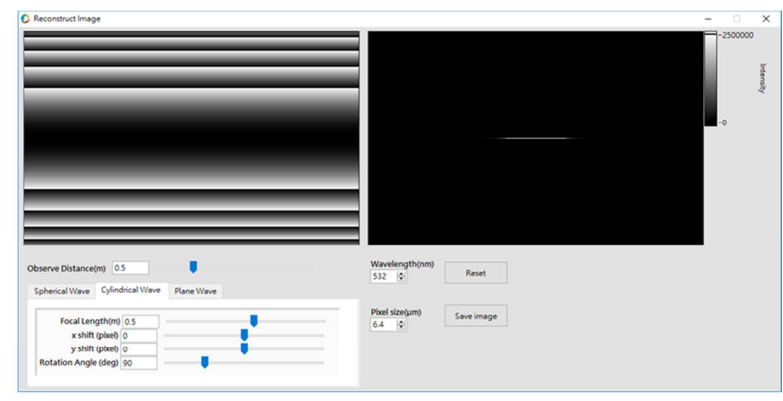

(b)

Figure 11. (a) LabVIEW based software GUI of wavefront modulation, and (b) GUI of wavefront modulation simulation.

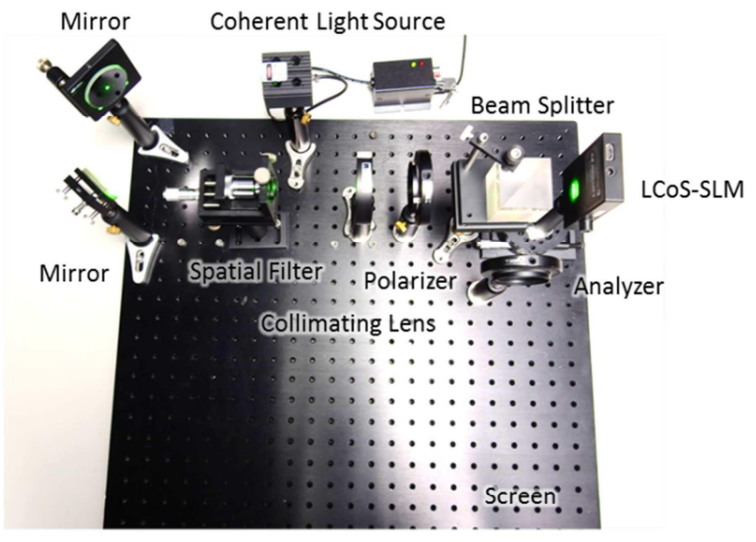

(a)

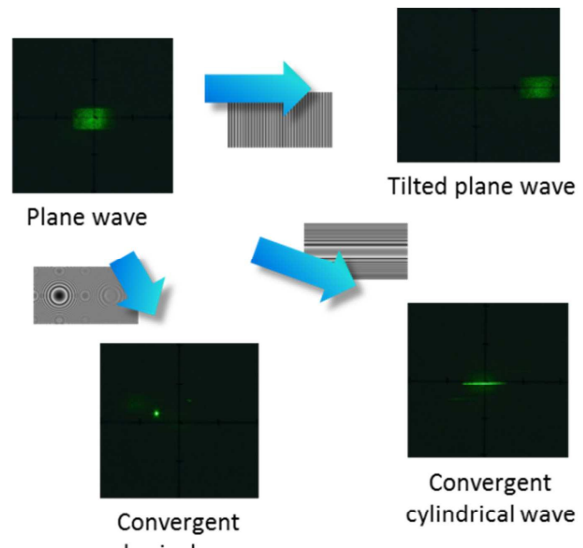

(b)

Figure 12. (a) Experiment setup photo of wavefront modulation, and (b) The diffraction image results at different diffraction pattern.

\subsection{Phase Shifting Digital Holography (PSDL)}

The objective of this experiment is to help students understand the operational principles of phase-shifting digital holography, as well as its device structure and method of calculating the amplitude and phase of the object wave. Furthermore, to help students learn how to use the near-field diffraction theory to verify the calculated outcome. This experiment for LCoS-SLM is Phase Shifting Digital Holography (PSDH). An object wave can be recorded by recording one set interference pattern. In PSDH, we record 4 interference patterns each has different phase shift $\left(0^{\circ}, 90^{\circ}, 180^{\circ}\right.$, $270^{\circ}$ ) in reference wave. By the following equation we can get the original wave information [18]

$$
O(x, y)=\frac{1}{4|R|}\left[\left(I_{0}-I_{\pi}\right)+i\left(I_{\pi} / 2-I_{3 \pi / 2}\right)\right]
$$

where $R$ is the reference wave, $I$ is interference of object and reference wave, and subscript 0 means the reference interference, other value mean the phase shift in reference wave. Therefore, we use Michaelson interferometer again and SLM is used to give phase retardation to the reference wave by giving different gray level input. The LabVIEW based software GUI of PSDH and the GUI of reconstruction image is shown in Figure 13 (a) and Figure 13 (b), respectively. Compare with the traditional PSDH, we can exactly change the phase of plane wave with the resolution of 8 bit depth by using EDK software. The experiment setup photo of phase-shifting digital holography is shown in Figure 14 (a). The 
interference fringes are recorded by a CCD and these phase difference are from phase $0^{\circ}, 90^{\circ}, 180^{\circ}$, and $270^{\circ}$ as shown as Figure 14 (b). Then, we use the image reconstruction software to load these four image files and to do the reconstruction procedure and calculation. The sample image and the reconstruct image are shown in Figure 14 (c).

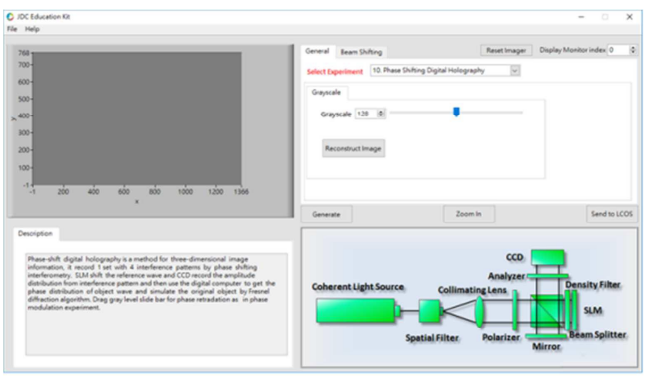

(a)

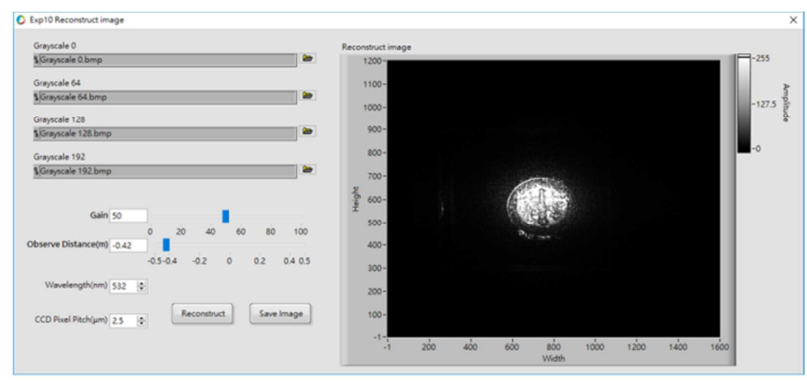

(b)

Figure 13. (a) LabVIEW based software GUI of phase shifting digital holography (PSDH), and (b) GUI of reconstruct image.

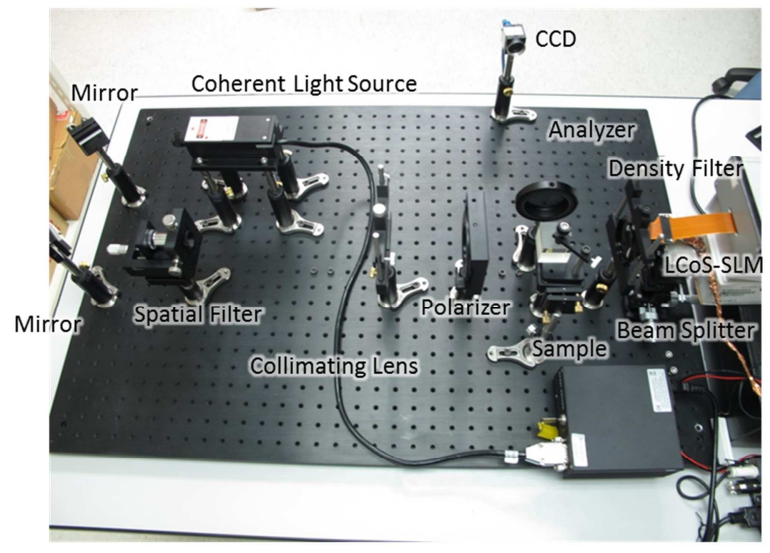

(a)
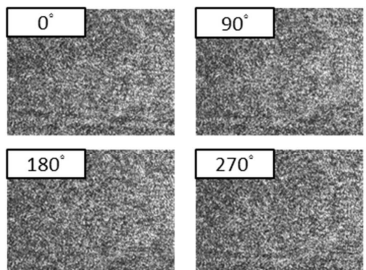

(b)
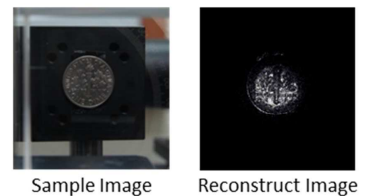

(c)

Figure 14. (a) Experiment setup photo of phase-shifting digital holography, (b) Interference fringes, phase difference from phase $0^{\circ}$, $90^{\circ}, 180^{\circ}$, and $270^{\circ}$, and (c) Sample image and reconstruct image from the above four interference fringes image.

\section{CONCLUSIONS}

In this paper, we have already developed multi-function and easy-to-use EDK software that was based on LabVIEW system. We would like to integrate these EDK software into our high reflectivity, high resolution LCoS-SLM to enhance the overall performance. Based on this LCoS-SLM (EDK), we provide a training platform which includes a series of optical theory and experiments to the university students. This EDK software not only provides a LabVIEW based operation software to produce CGH to generate some basic diffraction image or holographic image, but also provides simulation software to verity the experiment results simultaneously. Our LabVIEW based software can also simulate fundamental optics, wave optics, and Fourier optics. This simulation software not just helps students to realize the theory of Jones matrix and the relationship among of polarizer, retarder, and analyzer, but can simulate the interference, diffraction, and dispersion phenomenon. We also can use this simulation software to simulate the optical pattern and optical wavefront dynamically. However, we believe that a robust LCoS-SLM, operation software, simulation software, training system, and training course can help students to study the fundamental optics, wave optics, and Fourier optics more easily. Based on these fundamental knowledges, they could develop their unique skills and create their new innovations on the optoelectronic application in the future. 


\section{REFERENCES}

[1] Mu, Q., "Adaptive optics imaging system based on a high-resolution liquid crystal on silicon device," Optics Express, 14(18), 8013-8018 (2006)

[2] Chung, C.Y., Cho, K.C., Chang, C.C., Lin, C.H., Yen, W.C., Chen, S.J., “Adaptive-optics system with liquidcrystal phase- shift interferometer," Applied Optics, 45(15), 3409-3414 (2006).

[3] Tsai, C. W., Lyu, B. H., Wang, C., Hung, C. C., "Enhancing performance of LCoS-SLM as adaptive optics by using computer-generated holograms modulation software," Proceeding of SPIE 10233, Holography: Advances and Modern Trends, Prague, Czech Republic, 1023347 (2017).

[4] Kozacki, T., "Holographic display with tilted spatial light modulator," Applied Optics, 50(20), 3580-3588 (2011).

[5] Hsu, W. F., Weng, M. H., "Compact holographic projection display using liquid-crystal-on-silicon spatial light modulator," Materials, 9(9), 768 (2016)

[6] Lin, Y. C., Tu, H. Y. and Cheng, C. J., "Characterizing spatio-temporal phase variation of pixelated liquid crystal on silicon using digital holographic microscopy," Proceeding of Digital Holography and 3D Imaging, Shanghai, China, DTh1A.5 (2015).

[7] Krizek, P. and Hagen, G. M., "Spatial light modulators in fluorescence microscopy," Microscopy: Science, Technology, Applications and Education, Formatex Publisher, Spain, 1366-1377 (2010).

[8] Li, S., Wang, Y., Lu, Z., Ding, L., Du, P., Chen, Y., Zheng, Z., Ba, D., Dong, Y., Yuan, H., Bai, Z., Liu, Z., Cui, C., "High-quality near-field beam achieved in a high-power laser based on SLM adaptive beam-shaping system," Optics Express, 23(2), 681-689 (2015).

[9] McManamon, P.F., Bos, P.J., Escuti, M.J., Heikenfeld, J., Serati, S., Xie, H., Watson, E.A., "A Review of Phased Array Steering for Narrow-Band Electrooptical Systems," Proceedings of IEEE, 97(6), 1078-1096 (2009).

[10] Yang, H., Robertson, B., Wilkinson, P., and Chu, D., "Low-Cost CDC ROADM Architecture Based on Stacked Wavelength Selective Switches," Journal of Optical Communications and Networking, 9(5), 375-384 (2017).

[11] Milewski, G., Engström D., Bengtsson, J., "Diffractive optical elements designed for highly precise far-field generation in the presence of artifacts typical for pixelated spatial light modulators," Applied Optics, 46(1), 95105 (2007).

[12] Wilkinson, T. D., "Maskless Photolithography By Holographic Optical Projection," Imaging and Applied Optics, OSA Technical Digest (online), paper DW1B.3 (2014).

[13]Li, B., Gibson, J., Middendorf, J., Wang, Y., Zhang, S., "Comparison between LCOS projector and DLP projector in generating digital sinusoidal fringe patterns," Proceeding of SPIE 8839, Dimensional Optical Metrology and Inspection for Practical Applications II, 883908 (2013).

[14] Wang, C., Hsu, Y. C., Chan, S. H., "SLM-based education kit for wave optics," Proceeding of SPIE 9793, Education and Training in Optics and Photonics: ETOP 2015, Bordeaux, France, 979315 (2015).

[15] Wu, S. T., Yang, D. K., [Reflective Liquid Crystal Displays], WILEY SID Series in Display Technology, England (2001).

[16] Hecht, E., [Optics], Addison Wesley, San Francisco (2002).

[17] Yariv, A., Yeh, P., [Optical Waves in Crystals], WILEY INTER-SCIENCE, Canada (2003).

[18] Yamaguchi, I., Zhang, T., "Phase-shifting digital holography," Optics Letters, 22(16), 1268-1270 (1997). 